\title{
Blueberry supplementation reduces the blood lactate response to running in normobaric hypoxia but has no effect on performance in recreational runners
}

\author{
Jason P. Brandenburg* and Luisa V. Giles
}

\begin{abstract}
Background: Blueberries are concentrated with anthocyanins possessing antioxidant properties. As these properties counter fatigue, blueberry supplementation may improve performance and recovery, particularly in hypoxia, where oxidative stress is elevated.

Methods: This study examined the effects of blueberry supplementation on running performance, physiological responses, and recovery in normobaric hypoxia. Eleven experienced runners completed a 30-minute time-trial (TT) in normobaric hypoxia $\left(\% \mathrm{O}_{2}=15.5 \%\right)$ on separate days after supplementation with four days of blueberries (BLU) or four days of placebo (PLA). Heart rate (HR), oxygen saturation $\left(\mathrm{SaO}_{2}\right)$ and ratings of perceived exertion (RPE) were monitored during the $\Pi$. Blood lactate and fraction of exhaled nitric oxide $\left(F_{E N O}\right)$ were assessed pre- $T$, post- $T$, and during recovery.

Results: No significant differences were observed in the distance run during the $\Pi, \mathrm{HR}, \mathrm{SaO}_{2}$, and RPE. The post- $T$ increase in blood lactate was significantly lower in BLU than PLA $(p=0.036)$. Pre-TT and post-TT $F_{E N O}$ did not differ between conditions. Blood lactate recovery following the $\Pi$ was similar between conditions.

Conclusions: Four days of blueberry supplementation did not alter running performance or cardiovascular and perceptual responses in normobaric hypoxia. Supplementation lowered the blood lactate response to running, however, the significance of this finding is uncertain given the absence of an ergogenic effect.
\end{abstract}

Keywords: Polyphenols, Anthocyanins, Running, Endurance, Altitude, Nutrition

\section{Introduction}

The elevated metabolic requirements of exercise accelerate the production of reactive oxygen and nitrogen species (RONS) [1]. In small quantities, RONS function as signaling molecules that aid force production, help regulate muscle blood flow, and even trigger adaptations to exercise[2, 3]. However, when the accumulation of RONS is in excess, oxidative stress occurs, and this may

\footnotetext{
* Correspondence: jason.brandenburg@ufv.ca

Department of Kinesiology, School of Kinesiology, University of the Fraser Valley, 45190 Caen Avenue, BC V2R 0N3 Chilliwack, Canada
}

contribute to fatigue[3]. The contribution of oxidative stress to the development of fatigue is multifactorial and includes a reduced availability of nitric oxide (NO), which limits the capacity for vasodilation and blood flow[3]. Oxidative stress also disrupts calcium handling and sensitivity, consequently impairing force production[3]. The accumulation of RONS is influenced by the intensity and duration of exercise, as well as the environmental conditions in which the exercise is performed[4]. For example, when exercise is conducted in a hypoxic environment the degree of oxidative stress is heightened

(c) The Author(s). 2021 Open Access This article is licensed under a Creative Commons Attribution 4.0 International License, which permits use, sharing, adaptation, distribution and reproduction in any medium or format, as long as you give appropriate credit to the original author(s) and the source, provide a link to the Creative Commons licence, and indicate if changes were made. The images or other third party material in this article are included in the article's Creative Commons licence, unless indicated otherwise in a credit line to the material. If material is not included in the article's Creative Commons licence and your intended use is not permitted by statutory regulation or exceeds the permitted use, you will need to obtain permission directly from the copyright holder. To view a copy of this licence, visit http://creativecommons.org/licenses/by/4.0/. The Creative Commons Public Domain Dedication waiver (http://creativecommons.org/publicdomain/zero/1.0/) applies to the data made available in this article, unless otherwise stated in a credit line to the data. 
and is further implicated in the development of fatigue and decrements in performance $[5,6]$.

Considering the involvement of oxidative stress in exercise-induced fatigue and that higher levels of oxidative stress occur during exercise performed in hypoxia, strengthening an individual's antioxidant defenses, thereby preventing an excessive accumulation of RONS, may have an ergogenic effect on performance. Nutritionally, supplementation with polyphenol-rich fruits is one way to enhance an individual's antioxidant defenses [7, 8]. Polyphenols represent a class of compounds that have anti-inflammatory and antioxidant properties. Polyphenols are typically found in fruits and vegetables, and blueberries are considered to be an abundant source of polyphenols $[9$, 10]. Within blueberries, polyphenol compounds include phenolic acids and flavonoids, the most notable of which are anthocyanins[11]. From a health perspective, supplementation with blueberries has cardiovascular benefits including improved blood pressure, endothelial function, and arterial stiffness[12]. With respect to its ergogenic potential, acute blueberry supplementation mitigated the oxidative stress response to prolonged running[13]. However, as running performance was not assessed, it is unknown if this benefit translates into improved performance. Recent research suggests that although acute blueberry supplementation did not improve $8-\mathrm{km}$ running time-trial performance in normoxia, it did lessen the metabolic disturbances to running[14]. If blueberry supplementation lessens the metabolic disturbances to exercise in normoxia, it seems plausible that supplementation could aid performance in an environment in which the metabolic and oxidative stress disturbances to exercise are accentuated, as would be the case in hypoxia.

Currently, the ergogenic effect of acute supplementation with polyphenol-rich fruits on endurance performance is unclear due to mixed findings [15]. Moreover, the effects of anthocyanin-rich fruit supplementation (and specifically blueberries), on endurance performance and the physiological responses to exercise in hypoxia are not well understood due to the paucity of research. Consequently, the aim of the present study was to explore the influence of acute blueberry supplementation on 30-minute running time-trial performance and the physiological responses to running in normobaric hypoxia.

\section{Materials and Methods Study Design}

This study used a randomized, double-blind, placebocontrolled, crossover design with a 14-day washout period between experimental sessions. Participants visited the laboratory on three occasions. In the familiarization visit, participants performed the 30minute treadmill time trial (TT) in normobaric hypoxia
$\left(\mathrm{F}_{\mathrm{i}} \mathrm{O}_{2}=15.5 \%\right)$ and a graded running test to volitional exhaustion in normoxia to determine maximal oxygen consumption $\left(\mathrm{VO}_{2 \max }\right)$. The last two visits served as experimental sessions. During each experimental session, runners completed a 30-minute TT in normobaric hypoxia. Heart rate (HR), rating of perceived exertion (RPE), and oxygen saturation $\left(\mathrm{SaO}_{2}\right)$ were monitored throughout the TT. Blood lactate was assessed prior to, as well as 5, 15 and $30 \mathrm{~min}$ following the TT. Additionally, saliva and fraction of exhaled nitric oxide $\left(\mathrm{F}_{\mathrm{ENO}}\right)$ were sampled pre- and post-TT. In the four days before each experimental session, participants supplemented with 4 days of blueberry powder (BLU) or 4 days of a placebo powder (PLA). For each participant, sessions were conducted at the same time of day $( \pm 30 \mathrm{~min})$.

\section{Subjects}

Eleven recreational runners (4 males; 7 females) volunteered for this study (mean \pm SD: age $=28.4 \pm 7.5$ years; height $=170.9 \pm 9.0 \mathrm{~cm}$; body $\operatorname{mass}=65.6 \pm 9.7 \mathrm{~kg}$; $\left.\mathrm{VO}_{2 \max }=49.9 \pm 9.3 \mathrm{~mL} \cdot \mathrm{kg}^{-1} \cdot \mathrm{min}^{-1}\right)$. All participants were non-smokers; free from cardiovascular, respiratory, and oral/gum disease; and free from musculoskeletal injury. None of the participants had been exposed to altitude (natural or simulated equivalent to $\geq 1200 \mathrm{~m}$ above sea level) within the 3 months prior to the start of the study.

\section{Graded running test and $\mathrm{VO}_{2 \max }$}

During the familiarization session, the graded running test began at a treadmill speed considered comfortable by the participant (Alpine Runner, True Fitness Technology, St. Louis, MO). Treadmill gradient was increased by $2 \%$ every $2 \mathrm{~min}$ for the first $6 \mathrm{~min}$ of the test and then by $1 \%$ every 1 min until volitional exhaustion. Oxygen consumption was measured continuously throughout the test using a gas analysis system (TrueOne, Parvomedics, Sandy, Utah). $\mathrm{VO}_{2 \max }$ was determined as the highest 30-s average. All participants performed a 5minute warm-up at a self-selected speed before commencing the graded running test.

\section{Experimental Protocols}

Prior to the start of each experimental session, participants rinsed their mouth with distilled water and were fitted with a HR sensor and chest strap (Polar H7, Kempele, Finland). Participants were then moved into the altitude chamber (Altitudetech Inc, Kingston, ON, Canada) where they rested in a seated position for $10 \mathrm{~min}$. During this time each participant's food/activity log was reviewed to ensure compliance with study requirements.

Afterwards, participants provided saliva samples and pre-TT measures of $\mathrm{F}_{\mathrm{ENO}}$ and blood lactate were administered. Blood lactate was determined using a finger 
prick and handheld lactate analyzer (Lactate Pro; Arkray KDK Corp., Kyoto, Japan). The Lactate Pro analyzer, when compared to laboratory-based analyzers, has been shown to be both reliable (Coefficient of variation [CV] ranging from 2.7 to $5.7 \%$ ) and valid (SEE ranging from 0.5 to 1.1 ; Bias raging from $-0.6--1.7)$ [16-18]. $F_{\text {ENO }}$ was assessed using a handheld electrochemical analyzer (NIOX MINO, Aerocrine AB, Solna, Sweden) following previously established guidelines[19]. Once the pre-TT measures were completed, participants completed a 5minute self-paced warm-up followed by the 30-minute TT; both of which were performed on a non-motorized treadmill (Trueform Runner; Model: Performance; Connecticut, USA). Runners were instructed to run as far as possible during the $30 \mathrm{~min}$ (i.e. best effort). Runners were blinded to distance and running speed but were aware of the elapsed time. A fan was placed in front of the participant to simulate outdoor running conditions. A non-motorized treadmill was utilized for the TT because changes in running speed (i.e. pacing) are instantaneous, influenced by subconscious fatigue, and characteristic of over-ground running[20]. In experienced runners, like those in the present study, a TT on non-motorized treadmill has been shown to be reliable after a single familiarization session[16]. $\mathrm{HR}$ and $\mathrm{SaO}_{2}$, by fingertip pulse oximeter (MightySat, Masimo Corp, Irvine, $\mathrm{CA}$ ), were recorded throughout the TT while RPE (Borg 6-20 scale) was recorded every $5 \mathrm{~min}$.

Upon completion of the 30-minute TT, saliva was sampled, $\mathrm{F}_{\mathrm{ENO}}$ was assessed at $4 \mathrm{~min}$ post-TT, and blood lactate measurements were repeated at 5, 15, and $30 \mathrm{~min}$ post-TT. To assess lactate recovery, all post-TT values were normalized to the 5-minute post-TT value[21].

\section{Salivary Analyses}

Saliva was collected to measure cortisol, uric acid (UA), interleukin-6 (IL-6), and C-reactive protein (CRP). Salivary biomarkers were measured due to the ease and noninvasive nature of sample collection. The biomarkers analyzed in the present study have been shown to be valid and reliable indicators of the physiological stress to running[22, 23]. At each of the two saliva collection time points, two ml of saliva were collected using the passive drool method. Following saliva collection, samples were frozen at $-20{ }^{\circ} \mathrm{C}$ until analyses were conducted. Saliva samples were assayed using commercially available highly sensitive enzyme linked immunoassay kits (Salimetrics, PA, USA). Samples were analyzed in duplicate. The intra-assay CVs for the abovementioned saliva assays were below $5 \%$.

\section{Supplementation and Diet/Physical Activity Restrictions}

In the four days leading up to each experimental session, runners ingested either freeze-dried blueberry powder (BLU) (50/50 blend of Vaccinium virgatum/Vaccinium corymbosum, Oxygen Radical Absorbance Capacity [ORAC] $=831 \mu$ mole $\mathrm{TE} / \mathrm{g}$, Table 1 ) or placebo powder (PLA). PLA, manufactured to have a similar taste and appearance to the blueberry powder, was made from maltodextrin, fructose, dextrose, citric acid, malic acid, silicon dioxide (flow agent), xanthan gum, artificial colors, and artificial and natural flavours.

During the supplementation period, participants ingested 3, 24 g packets of blueberry or placebo powder per day (BLU $=\sim 500 \mathrm{~g}$ of fresh blueberries/day). Ingestion of the packets was distributed evenly throughout the day with the final packet ingested $2 \mathrm{~h}$ prior to the start of the session. For ingestion, the powder was mixed with about $250 \mathrm{ml}$ of water. The antioxidant and anthocyanin content of the blueberry powder used in the present study (Table 1) was assessed in the batch by a commercial laboratory (International Chemistry Testing, Milford, MA). Antioxidant activity was assessed using the Oxygen Radical Absorbance Capacity (ORAC) test based on the methods of $\mathrm{Ou}$ et al.[24]. The anthocyanin assay was based on the methods described by Lee et al.[25].

During each 4-day supplementation period participants were asked to limit the intake of other foods/ drinks high in polyphenols as well as refrain from taking other supplements and anti-inflammatory medications. Additionally, participants avoided caffeine and alcohol intake 8 and $24 \mathrm{~h}$ (respectively) prior to the start of each session. Participants also abstained from moderate to vigorous intensity physical activity within $24 \mathrm{~h}$ of each session. To facilitate this, participants completed a 4-day food and physical activity diary before the first experimental session and were instructed to replicate this diary before the second experimental session.

\section{Statistical analysis}

Normality of the data was evaluated using the ShapiroWilk test. Mauchly test of sphericity was performed to assess the homogeneity of data. Where violations were present, Greenhouse-Geisser adjustments were made. Between-supplement differences in 30-min running distance, average $\mathrm{HR}$, maximum $\mathrm{HR}$, average $\mathrm{SaO}_{2}$, RPE, pre-TT salivary biomarkers, and pre- to post-TT change in salivary biomarkers were analyzed using a paired $\mathrm{t}$ test. Differences in $\mathrm{F}_{\mathrm{ENO}}$ and blood lactate were analyzed using a repeated measures ANOVA with 2 supplement conditions by 2 time points ( 1 pre- and 1 post-TT measure). Differences in blood lactate recovery were analyzed using a repeated measures ANOVA with 2 supplement conditions by 3 time points (5-min, 15-min, and 30-min post-TT measures). Post hoc analysis was performed using t-tests with a Bonferroni correction. Statistical significance was set at $p<0.05$. To estimate the magnitude of the effects from supplementation, Cohen's d effect 
Table 1 Nutrient composition of the freeze-dried blueberry and placebo powders

\begin{tabular}{lll}
\hline & Freeze-dried blueberry powder (per 24 g packet) & Placebo powder (per 24 g packet) \\
\hline Energy (kcal) & 96 & 94 \\
Total Carbohydrates (g) & 21.8 & 22.7 \\
Protein (g) & 1 & 0.19 \\
Fat (g) & 0.6 & 0.02 \\
Dietary Fiber (g) & 5.1 & 0 \\
Vitamin C (mg) & 3.9 & 0 \\
Phenolics (mg) & 811 & 0 \\
Anthocyanins (mg) & 336 & 0 \\
\hline
\end{tabular}

sizes (ES) were calculated with the magnitude of effects considered small (0.20-0.49), moderate (0.50-0.79) and large $(>0.80)$. Additionally, Pearson correlation coefficients were used to explore relationships between the study variables. Data are presented as mean \pm SD. All statistical analyses were conducted using SPSS v 20.0 (SPSS Inc., Chicago, IL, USA).

\section{Results}

No difference in the distance achieved during the 30minute TT in normobaric hypoxia was observed between supplement conditions (Table 2). Heart rate, $\mathrm{SaO}_{2}$, and RPE responses during the TT were not different between the two supplement conditions (Table 2).

Pre- and post-TT $F_{E N O}$ levels were not different between the two supplement conditions (Table 3). In both conditions, blood lactate significantly increased following the 30-min TT $(p<0.001)$ with the increase in PLA being significantly greater than BLU $(p=0.036)$ (Table 3$)$. Post-hoc comparisons indicated the blood lactate concentration following the TT was significantly lower in BLU than PLA $(p=0.02)$. A Cohen's $\mathrm{d}$ showed a moderate effect size $(d=0.76)$ for the attenuated post-TT increase in blood lactate following BLU. Additionally, 8 of

Table 2 Performance and physiological variables during the 30minute time trial completed in normobaric hypoxia

\begin{tabular}{lll}
\hline Variable & PLA & BLU \\
\hline 30-minute time trial $(\mathrm{km})$ & & \\
0-15 min split & $2.21 \pm 0.31$ & $2.22 \pm 0.38$ \\
15-30 min split & $2.26 \pm 0.32$ & $2.24 \pm 0.33$ \\
Total distance & $4.46 \pm 0.62$ & $4.47 \pm 0.69$ \\
Heart Rate (HR) & & \\
Average HR during TT & $176.3 \pm 13.7$ & $175.3 \pm 12.7$ \\
Maximum HR during $\Pi$ & $187.3 \pm 15.1$ & $186.6 \pm 13.4$ \\
\%SaO & & \\
30-minute average during TT & $89.3 \pm 1.4$ & $89.2 \pm 2.1$ \\
Rating of Perceived Exertion (RPE) & & \\
Maximum RPE during $\Pi$ & $18.1 \pm 1.4$ & $18.5 \pm 1.2$ \\
\hline
\end{tabular}

the 11 runners experienced less of an increase in blood lactate in response to BLU (Fig. 1). There was no significant difference in blood lactate recovery at $15 \mathrm{~min}$ as well as 30 min post-TT, although a moderate effect size was observed at $30 \mathrm{~min}(d=0.51)$ (Table 3$)$.

No significant pre-TT differences were observed in any of the salivary biomarkers between PLA and BLU (Table 3). However, a Cohen's d showed a moderate effect size $(d=0.68)$ for lower pre-exercise cortisol levels in response to BLU. The pre- to post-TT increases in cortisol and IL-6 were significant $(p<0.001$ and $p=$

Table 3 Blood lactate, $F_{\text {ENO, }}$ and salivary biomarker results before and after the 30-minute time trial

\begin{tabular}{lrc}
\hline Variable & PLA & \multicolumn{1}{l}{ BLU } \\
\hline Exhaled Nitric Oxide $(\mathrm{ppb})$ & $18.5 \pm 8.2$ & $18.7 \pm 6.1$ \\
Pre-exercise & $17.2 \pm 6.4$ & $17.2 \pm 4.2$ \\
Post-exercise & & \\
Blood Lactate $\left(\mathrm{mmol} \cdot \mathrm{I}^{-1}\right)$ & $1.5 \pm 0.4$ & $1.5 \pm 0.4$ \\
Pre-TT & $8.9 \pm 2.7$ & $7.2 \pm 1.9^{*}$ \\
5-minute post- $T$ &
\end{tabular}

Blood Lactate Recovery (\% of 5-min post-TT)

Recovery at 15 min post- $T \quad 57.5 \pm 16.7 \quad 56.7 \pm 9.8$

Recovery at 30 min post- $T$ T $\quad 37.2 \pm 12.1 \quad 32.5 \pm 5.1$

Salivary Cortisol $(\mu \mathrm{g} / \mathrm{dL})$

$\begin{array}{lll}\text { Pre-TT } & 0.23 \pm 0.11 & 0.16 \pm 0.06\end{array}$

$\begin{array}{lll}\text { Post-TT } & 0.53 \pm 0.34 & 0.52 \pm 0.27\end{array}$

Salivary Uric Acid $(\mathrm{mg} / \mathrm{dL})$

$\begin{array}{lll}\text { Pre-TT } & 3.11 \pm 0.81 & 2.72 \pm 0.89\end{array}$

$\begin{array}{lll}\text { Post-TT } & 3.04 \pm 1.26 & 2.83 \pm 0.93\end{array}$

Salivary IL-6 $(\mathrm{pg} / \mathrm{mL})$

$\begin{array}{lrr}\text { Pre-TT } & 8.9 \pm 6.4 & 7.4 \pm 4.2\end{array}$

$\begin{array}{lll}\text { Post-TT } & 23.2 \pm 9.2 & 24.3 \pm 19.8\end{array}$

Salivary CRP $(p g / d L)$

$\begin{array}{lll}\text { Pre-TT } & 6162 \pm 5547 & 6504 \pm 8389\end{array}$

Post-TT 14,796 $\pm 20,641 \quad 10,771 \pm 13,987$

${ }^{*}=$ Significantly different than PLA at same time point $(p=0.02)$ 


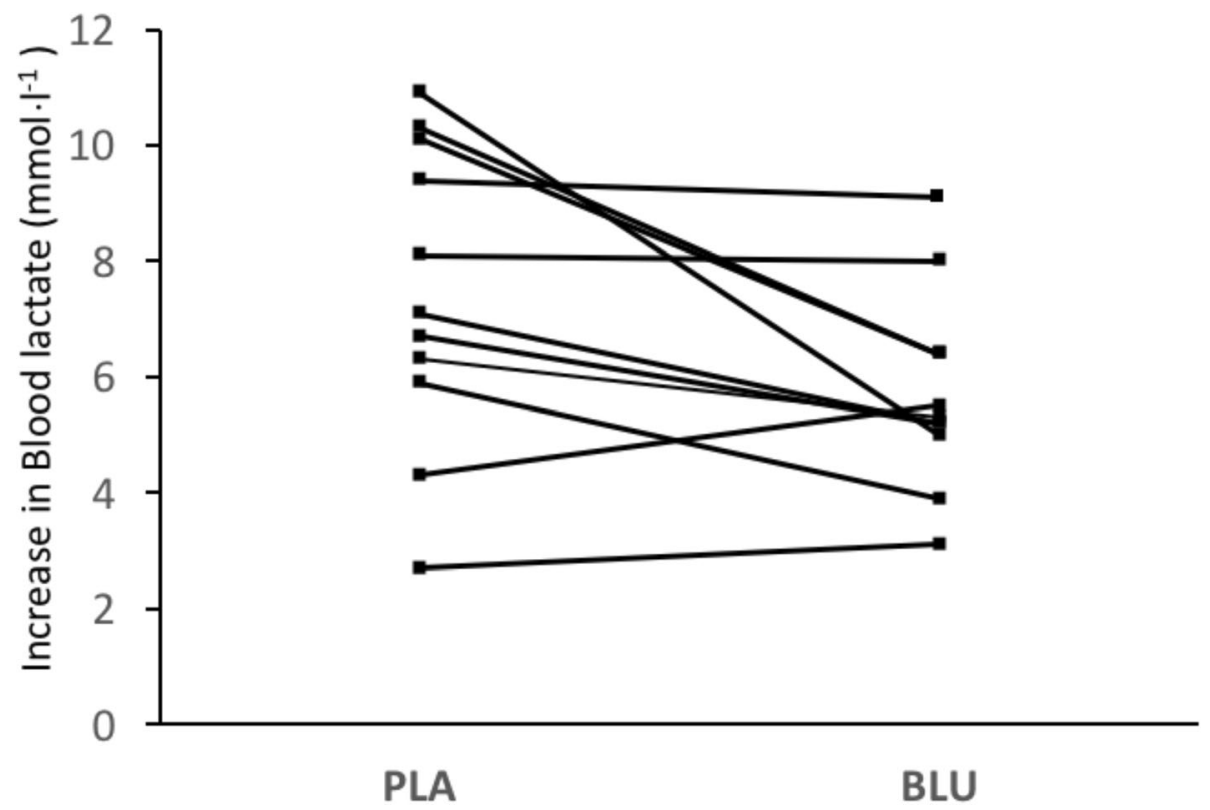

Fig. 1 Individual increases in blood lactate concentrations (pre-TT to 5-minute post-TT) in response to PLA and BLU

0.005, respectively) with no differences between the two supplement conditions. For UA and CRP, pre- to postTT changes were not significant.

A moderate negative relationship was observed between the PLA vs. BLU difference in pre-TT $F_{E N O}$ and the PLA vs. BLU difference in the pre-TT to post-TT increase in blood lactate concentration $(\mathrm{r}=-0.50, p=$ $0.069)$.

\section{Discussion}

The present study examined the effect of acute blueberry supplementation on running performance and physiological responses in normobaric hypoxia. Four days of supplementation had no influence on 30-minute TT performance. However, although most of the physiological variables remained unaffected following supplementation, the post-TT increase in blood lactate was attenuated following blueberry supplementation.

Following the 30-minute TT, blood lactate was 1.7 mmol $\cdot l^{-1}$ or $\sim 15 \%$ lower in BLU than PLA (Table 3 ). When compared to the CV associated with the Lactate Pro analyzer $(2.7-5.7 \%)$ [16-18], the $15 \%$ reduction in the blood lactate response points to an intervention effect due to acute blueberry supplementation. This interpretation is reinforced considering 8 of the 11 participants experienced a mitigated post-TT increase in blood lactate following BLU (Fig. 1). The attenuated blood lactate response to the 30-minute TT following BLU is similar to that observed following an $8-\mathrm{km}$ running TT in normoxia in response to a 4-day blueberry supplementation protocol[14]. These findings also mirror the $4 \%$ reduction (not significant) in blood lactate levels in highly-trained cyclists following completion of a TT to exhaustion in normobaric hypoxia $\left(\mathrm{F}_{\mathrm{i}} \mathrm{O}_{2}=\sim\right.$ $17 \%)$ after supplementation with pomegranate extract[26]. In contrast, blood lactate responses to low and moderate intensity cycling in normobaric hypoxia $\left(\mathrm{F}_{\mathrm{i}} \mathrm{O}_{2}=\sim 15 \%\right)$ were not altered after 7 days of supplementation with black currant extract[27]. The contradiction between these findings and those of the present study could be due to differences between the supplementation protocols. In the current study, participants received $336 \mathrm{mg}$ of anthocyanins at each point of ingestion $(1008 \mathrm{mg} /$ day); with the final dose ingested $2 \mathrm{~h}$ prior to testing. In the study by Willems et al.[28], participants received $105 \mathrm{mg}$ of anthocyanins at each intake (210 $\mathrm{mg} /$ day); with the timing of the final supplement greater than $2 \mathrm{~h}$ before testing. Physiologically, both the dose of anthocyanins and timing of the last dose influence endothelial function; thought to be principal mechanism underlying the ergogenic effect of anthocyaninrich fruit supplementation [15]. With respect to dose, acute improvements in endothelial function have been shown to linearly increase in response to an increasing dose of anthocyanins (up to $310 \mathrm{mg}$ )[9]. Further, increases in endothelial function following anthocyaninrich fruit supplementation peak 1-2 h post-ingestion[9]. With these factors in mind, it seems plausible the greater dose of anthocyanins used in the current study combined with the timing of the last dose relative to exercise (compared to those used by Willems et al.) could have yielded greater improvements endothelial function. 
If so, improvements in endothelial function may have resulted in increased muscle blood flow, subsequently improving lactate clearance and accounting for the lower post-TT blood lactate values following BLU.

Accounting for the improvements in endothelial function following blueberry supplementation is an increase in NO bioavailability [9]. In the current study, $\mathrm{F}_{\mathrm{ENO}}$ was used as an indirect marker of $\mathrm{NO}$ availability. To this end, significant increases in $\mathrm{F}_{\mathrm{ENO}}$ have been observed In response to acute beetroot juice supplementation [24, 25]. Accompanying the increases in $\mathrm{F}_{\mathrm{ENO}}$ were elevated levels of plasma nitrite and nitrate (precursors of NO production); thus suggestive of elevated $\mathrm{NO}$ availability in the blood[29]. Considering acute blueberry supplementation also increases NO bioavailability [9], albeit not by the same mechanisms, it was thought that blueberries would have a similar effect on $\mathrm{F}_{\mathrm{ENO}}$ as nitratecontaining supplements, such as concentrated beetroot juice. However, four days of blueberry supplementation had no effect on $\mathrm{F}_{\mathrm{ENO}}$ as evidenced by the similar preTT values. Consequently, it is uncertain if blueberry supplementation had any impact on NO availability, raising questions about the contribution of altered blood flow to the lower blood lactate response. Despite this, there was a moderate negative relationship $(\mathrm{r}=-0.50, p=0.06)$ between the BLU vs. PLA difference in pre-TT $F_{\mathrm{ENO}}$ and the BLU vs. PLA difference in the pre-TT to post-TT increase in blood lactate. This suggests that those runners who experienced increases in $\mathrm{F}_{\mathrm{ENO}}$ in response to blueberry supplementation (in comparison to PLA) tended to experience less of an increase in blood lactate following the TT. Thus, as with other forms of polyphenolrich supplementation, this relationship indicates the physiological response to blueberry supplementation seems to be individualized (i.e. responders and nonresponders)[29]. Two factors thought to contribute to the individual responses to supplementation are training status and diet (i.e. dietary intake of large amounts of polyphenols) $[29,30]$. In the context of the present study, the recreational status of the participants along with the restriction of other polyphenol-rich foods/drinks during the 4-day supplementation periods may have contributed to the uniform blood lactate response to the 30-minute TT (i.e. 8 of 11 participants experienced a smaller increase). Although consistent, any ergogenic benefits from this response are uncertain as there were no improvements in 30-minute TT performance.

The absence of any performance advantage following blueberry supplementation is in agreement with recent findings. In a simulated altitude comparable to that of the present study, $10-\mathrm{km}$ treadmill TT performance in trained runners was not significantly altered following a single dose of concentrated beetroot juice $\left(\mathrm{F}_{\mathrm{i}} \mathrm{O}_{2}=\right.$ 15.4\%)[30]. Similarly, after seven days of blackcurrant extract supplementation recreational cyclists experienced no improvement in a $16.1 \mathrm{~km}$ cycling TT in normobaric hypoxia $\left(\mathrm{F}_{\mathrm{i}} \mathrm{O}_{2}=\sim 15 \%\right)$ [27]. When considered collectively, the lack of any significant performance improvement in these studies, and in the present study, may be due to the intensity of exercise task and/or the degree of hypoxia. With respect to intensity, when the duration of the running task was reduced to $1500 \mathrm{~m}$ (and therefore intensity increased) supplementation with concentrated beetroot juice significantly improved performance in normobaric hypoxia $\left(\mathrm{F}_{\mathrm{i}} \mathrm{O}_{2}=\sim 15 \%\right)$ [29]. Furthermore, significant improvements in $1500 \mathrm{~m}$ running time, but not $10 \mathrm{~km}$ running time, were observed at sea level following supplementation with concentrated beetroot juice[31]. The completion of higher intensity exercise, like a $1500 \mathrm{~m}$ running TT when compared to the 30minute TT of the present study or even a $16.1 \mathrm{~km}$ cycling TT, is more limited by the delivery of $\mathrm{O}_{2}$ to sustain the elevated metabolic requirements of the exercising muscle[2]. Consequently, higher intensity exercise is more likely to benefit following a supplement that improves muscle blood flow via an increase in NO. In the current study, it seems reasonable to suggest the lack of any ergogenic effect following blueberry supplementation may have been due to the relatively modest intensity of the 30-minute TT and that the performance of it was not limited by $\mathrm{O}_{2}$ delivery.

\section{Conclusions}

To conclude, four days of blueberry supplementation attenuated the increase in blood lactate to a 30-minute running time trial in normobaric hypoxia. Despite this, there was no improvement in running performance. Consequently, the use of acute blueberry supplementation as an ergogenic aid for endurance performance in hypoxia is not supported.

\section{Abbreviations}

BLU: Blueberry supplementation condition; PLA: Placebo supplementation condition; TT: Time-trial; HR: Heart Rate; RPE: Rating of Perceived Exertion; $\mathrm{SaO}_{2}$ : $\mathrm{O} 2$ saturation; $\mathrm{F}_{\mathrm{ENO}}$ : fraction of exhaled nitric oxide; $\mathrm{F}_{\mathrm{O}} \mathrm{O}_{2}$ : Fraction of inspired $\mathrm{O}_{2} ; \mathrm{VO}_{2 \text { max }}$ : Maximal $\mathrm{O}_{2}$ consumption; UA: Uric acid; IL-6: Interleukin6; CRP: C-reactive protein; ORAC: $\mathrm{O}_{2}$ Radical Absorbance Capacity;

$\mathrm{CV}$ : Coefficient of Variation

\section{Authors' contributions}

JPB contributed to the design of the study, to data collection, analysis, and interpretation, as well as to the development of the manuscript. LVG contributed to the design of the study, analysis, and interpretation, as well as to the development of the manuscript. All authors have read and approved the final version of the manuscript.

\section{Funding}

This project was supported by a grant from the U.S. Highbush Blueberry Council (USHBC). The USBC was not involved in the design of the study, data collection, data analysis, data interpretation, nor in the writing of the manuscript. 


\section{Availability of data and materials}

The datasets generated and/or analyzed during the current study are not publicly available but are available from the corresponding author on reasonable request.

\section{Declarations}

\section{Ethics approval and consent to participate}

The study was conducted in accordance with the Declaration of Helsinki and the protocols were approved by the University of the Fraser Valley Human Research Ethics Board.

\section{Consent for publication}

All authors approved the final version of the paper.

\section{Competing interests}

The authors declare that they have no competing interests.

\section{Received: 23 November 2020 Accepted: 12 March 2021}

Published online: 30 March 2021

\section{References}

1. Ferreira LF, Reid MB. Muscle-derived ROS and thiol regulation in muscle fatigue. J Appl Physiol. 2008;104(3):853-860. doi:https://doi.org/10.1152/ja pplphysiol. 00953.2007. PMID: 18006866

2. Kashi DS, Shabir A, Da Boit M, Bailey SJ, Higgins M. The efficacy of administering fruit-derived polyphenols to improve health biomarkers, exercise performance and related physiological responses. Nutrients. 2019; 11(10):2389. doi:https://doi.org/10.3390/nu11102389. PMID: 31591287.

3. Reid MB. Redox interventions to increase exercise performance. J Physiol. 2016;594(18): 5125-5133. doi:https://doi.org/10.1113/JP270653. PMID: 26584644

4. Vinña J, Gomez-Cabrera M-C, Lloret A, Marquez R, Miñana JB, Pallardó FV, et al. Free radicals in exhaustive physical exercise: mechanism of production, and protection by antioxidants. IUBMB Life. 2000;50(4):271-277. doi: 713803729. PMID: 11327321

5. Ribon A, Pialoux V, Saugy JJ, Rupp T, Faiss R, Debevec T, et al. Exposure to hypobaric hypoxia results in higher oxidative stress compared to normobaric hypoxia. Respir Physiol Neurobiol. 2016;223:23-27. doi:10.1016/j. resp.2015.12.008. PMID: 26732282

6. McGinnis G, Kliszczewiscz B, Barberio M, Ballmann C, Peters B, Slivka D, et al. Acute hypoxia and exercise-induced blood oxidative stress. Int J Sport Nutr Exerc Metab. 2014;24(6):684-693. doi:https://doi.org/10.1123/ijsnem.2013-01 88. PMID: 24667140

7. Myburgh $\mathrm{KH}$. Polyphenol supplementation: Benefits for exercise performance or oxidative stress? Sport Med. 2014;44(S1):57-70. doi:https:// doi.org/10.1007/s40279-014-0151-4. PMID: 24791917.

8. Bowtell J, Kelly V Fruit-Derived Polyphenol Supplementation for Athlete Recovery and Performance. Sport Med. 2019:49(S1), 3-23. doi:https://doi. org/10.1007/s40279-018-0998-x. PMID: 30671906

9. Rodriguez-Mateos A, Rendeiro C, Bergillos-Meca T, Tabatabaee S, George TW, Heiss $C$, et al. Intake and time dependence of blueberry flavonoidinduced improvements in vascular function: A randomized, controlled, double-blind, crossover intervention study with mechanistic insights into biological activity. Am J Clin Nutr. 2013; 98(5),1179-1191. doi:https://doi. org/10.3945/ajcn.113.066639. PMID: 24004888.

10. Rodriguez-Mateos A, Cifuentes-Gomez T, Tabatabaee S, Lecras C, Spencer IPE. Procyanidin, anthocyanin, and chlorogenic acid contents of highbush and lowbush blueberries. J Agric Food Chem. 2012; 60(23):5772-5778. doi: https://doi.org/10.1021/jf203812w. PMID: 22175691

11. Taruscio TG, Barney DL, Exon J. Content and Profile of Flavanoid and Phenolic Acid Compounds in Conjunction with the Antioxidant Capacity for a Variety of Northwest Vaccinium Berries. J Agric Food Chem. 2004; 52(10): 3169-3176. doi:https://doi.org/10.1021/jf0307595. PMID: 15137871.

12. Johnson SA, Figueroa A, Navaei N, Wong A, Kalfon R, Ormsbee LT, et al. Daily blueberry consumption improves blood pressure and arterial stiffness in postmenopausal women with pre- and stage 1-hypertension: A randomized, double-blind, placebo-controlled clinical trial. J Acad Nutr Diet. 2015; 15(3):369-377. doi:10.1016/j.jand.2014.11.001. PMID: 25578927
13. McAnulty LS, Nieman DC, Dumke CL, Shooter LA, Henson DA, Utter AC, et al. Effect of blueberry ingestion on natural killer cell counts, oxidative stress, and inflammation prior to and after $2.5 \mathrm{~h}$ of running. Appl Physiol Nutr Metab. 2011; 36(6):976-984. doi:https://doi.org/10.1139/h11-120. PMID: 22111516.

14. Brandenburg JP, Giles LV. Four days of blueberry powder supplementation lowers the blood lactate response to running but has no effect on time-trial performance. Int J Sport Nutr Exerc Metab. 2019;1-7. doi:https://doi.org/1 0.1123/ijsnem.2019-0040. PMID: 31629347.

15. Mason SA, Trewin AJ, Parker L, Wadley GD. Antioxidant supplements and endurance exercise: Current evidence and mechanistic insights. Redox Biol. 2020;35:101471. doi:https://doi.org/10.1016/j.redox.2020.101471.

16. Pyne DB, Boston T, Martin DT, Logan A. Evaluation of the Lactate Pro blood lactate analyser. Eur J Appl Physiol. 2000;82(1-2):112-6. doi:https://doi.org/1 $0.1007 / \mathrm{s} 004210050659$

17. Tanner RK, Fuller KL, Ross ML. Evaluation of three portable blood lactate analysers: Lactate Pro, Lactate Scout and Lactate Plus. Eur Appl Physiol. 2010;109(3):551-9. doi:https://doi.org/10.1007/s00421-01 0-1379-9.

18. Baldari C, Bonavolontà V, Emerenziani GP, Gallotta MC, Silva AJ, Guidetti L. Accuracy, reliability, linearity of Accutrend and Lactate Pro versus EBIO plus analyzer. Eur J Appl Physiol. 2009;107(1):105-11. doi:https://doi.org/10.1007/ s00421-009-1107-5.

19. ATS/ERS recommendations for standardized procedures for the. online and offline measurement of exhaled lower respiratory nitric oxide and nasal nitric oxide. Am J Respir Crit Care Med. 2005;171:912-30. doi:https://doi. org/10.1164/rccm.200406-710ST.

20. Stevens CJ, Hacene J, Sculley DV, Taylor L, Callister R, Dascombe B. The Reliability of Running Performance in a $5 \mathrm{~km}$ Time Trial on a Non-motorized Treadmill. Int J Sports Med. 2015;36(9):705-709. doi:https://doi.org/10.1055/ s-0034-1398680. PMID: 25790087

21. Wiewelhove T, Schneider C, Schmidt A, Döweling A, Meyer T, Kellmann M et al. Active Recovery After High-Intensity Interval-Training Does Not Attenuate Training Adaptation. Front Physiol. 9:415. doi:https://doi.org/10.33 89/fphys.2018.00415. PMID: 29720949

22. Papacosta E, Nassis GP. Saliva as a tool for monitoring steroid, peptide and immune markers in sport and exercise science. Vol. 14, Journal of Science and Medicine in Sport. 2011; 14(5), 424-434. doi:https://doi.org/10.1016/j.jsa ms.2011.03.004. PMID: 21474377.

23. Tauler P, Martinez S, Moreno C, Martínez P, Aguilo A. Changes in salivary hormones, immunoglobulin $\mathrm{A}$, and $\mathrm{C}$-reactive protein in response to ultraendurance exercises. Appl Physiol Nutr Metab. 2014;39(5):560-565. doi: https://doi.org/10.1139/apnm-2013-0466. PMID: 24766238.

24. Ou B, Hampsch-Woodill M, Prior RL. Development and validation of an improved oxygen radical absorbance capacity assay using fluorescein as the fluorescent probe. J Agric Food Chem. 2001;49(10):4619-26.

25. Lee J, Durst RW, Wrolstad RE. Determination of total monomeric anthocyanin pigment content of fruit juices, beverages, natural colorants, and wines by the pH differential method: Collaborative study. J AOAC Int. 2005;88(5):1269-78. PMID: 16385975.

26. Crum EM, Che Muhamed AM, Barnes M, Stannard SR. The effect of acute pomegranate extract supplementation on oxygen uptake in highly-trained cyclists during high-intensity exercise in a high altitude environment. J Int Soc Sports Nutr. 2017;14:14. doi:https://doi.org/10.1186/s12970-017-0172-0. PMID: 28572749

27. Willems M, Sahin M, Berendsen T, Cook M. Effect of New Zealand Blackcurrant Extract on Cycling Performance and Substrate Oxidation in Normobaric Hypoxia in Trained Cyclists. Sports. 2019:7(3):67. doi:https://doi. org/10.3390/sports7030067. PMID: 30884866

28. MacLeod KE, Nugent SF, Barr SI, Koehle MS, Sporer BC, Maclnnis MJ. Acute Beetroot juice supplementation does not improve cycling performance in normoxia or moderate hypoxia. Int J Sport Nutr Exerc Metab. 2015;25(4):359-366. doi:https://doi.org/10.1123/ijsnem.2014-0129. PMID: 25811674.

29. Shannon OM, Duckworth L, Barlow MJ, Woods D, Lara J, Siervo M, et al. Dietary nitrate supplementation enhances high-intensity running performance in moderate normobaric hypoxia, independent of aerobic fitness. Nitric Oxide. 2016; 9:63-70. doi:https://doi.org/10.1016/j.niox.2016.08. 001. PMID: 27553127.

30. Arnold JT, Oliver SJ, Lewis-Jones TM, Wylie LJ, Macdonald JH. Beetroot juice does not enhance altitude running performance in well-trained athletes. 
Appl Physiol Nutr Metab. 2015;40(6):590-595. doi:https://doi.org/10.1139/a pnm-2014-0470. PMID: 25942474.

31. Shannon OM, Barlow MJ, Duckworth L, Williams E, Wort G, Woods D, et al. Dietary nitrate supplementation enhances short but not longer duration running time-trial performance. Eur J Appl Physiol. 2017;117(4):775-785. doi: https://doi.org/10.1007/s00421-017-3580-6. PMID: 28251402.

\section{Publisher's Note}

Springer Nature remains neutral with regard to jurisdictional claims in published maps and institutional affiliations.

Ready to submit your research? Choose BMC and benefit from:

- fast, convenient online submission

- thorough peer review by experienced researchers in your field

- rapid publication on acceptance

- support for research data, including large and complex data types

- gold Open Access which fosters wider collaboration and increased citations

- maximum visibility for your research: over $100 \mathrm{M}$ website views per year

At $B M C$, research is always in progress.

Learn more biomedcentral.com/submissions 